\title{
Potentielle infection nosocomiale à Mycobacterium chimaera
}

\section{Barbara Hasse, en collaboration avec la Task Force M. Chimaera*}

PD Dr méd., Klinik für Infektionskrankheiten und Spitalhygiene, UniversitätsSpital Zurich

* Erik C. Böttger, Zurich; Simon Costabile, Zurich; Samuel Erny, Berne; Céline Gardiol, Berne; Achim Häussler, Zurich; Peter Keller, Zurich; Daniel Koch, Berne; Virginie Masserey, Berne; Rafael Moreno, Berne; Hugo Sax, Zurich; Matthias Schlegel, St-Gall; Bettina Schulthess, Zurich; Rami Sommerstein, Berne;

Thomas Suter, Berne; Markus Wälti, Berne; Andreas Widmer, Bâle

Lorsqu'un patient développe une infection d'origine indéterminée, une endocardite à hémocultures négatives ou des symptômes de maladie systémique des mois ou des années après une intervention chirurgicale à cœur ouvert, une infection à Mycobacterium chimaera devrait être suspectée et recherchée. Ce document résume les situations où de plus amples investigations devraient être réalisées.

\section{Situation initiale}

A l'Hôpital universitaire de Zurich (USZ) et aux Hôpitaux universitaires de Bâle et Liestal (USB), à ce jour 10 patients ont été diagnostiqués avec une infection grave et disséminée à Mycobacterium chimaera après une opération à cœur ouvert. Cette mycobactérie non tuberculeuse a probablement été transmise aux patients durant l'intervention par aérosolisation d'eau contaminée dans le champ opératoire provenant d'un système de régulation de température de la firme Sorin (à présent Livanova) de Type 3T (Heater Cooler Devices, HCDs) [1]. L'Office fédéral de la santé publique (OFSP) et Swissmedic en ont informé le public et les hôpitaux le 14 juillet 2014 [2, 3]. Entre-temps d'autres HCDs contaminés ont été découverts en Suisse et dans d'autres pays [4].

Les LivaNova 3T HCDs ont été contaminés soit lors de leur fabrication soit dans les hôpitaux par de l'eau du robinet. Plus de 70 patients infectés par $M$. chimaera ont été diagnostiqués partout dans le monde. Il est possible qu'il existe (même en Suisse) d'autres patients infectés mais non encore diagnostiqués. Le diagnostic est difficile à poser, car les premiers symptômes ne sont pas spécifiques, la bactérie est difficile à cultiver et le temps d'incubation peut durer jusqu'à 5 ans. Nombreux de ces cas ont été en premier lieu classifiés comme fièvre d'origine indéterminée (FUO) et quelques-uns ont reçu un diagnostic erroné de sarcoïdose et ont été traités avec des cortico-stéroïdes. Le diagnostic est d'autant plus difficile que les manifestations extracardiaques de la maladie apparaissent souvent avant les manifestations cardiaques (infection osseuse, pancytopénie, choriorétinite, atteintes du foie et des reins).

Le présent document devrait contribuer à reconnaître le plus vite possible les patients concernés, vu que, malgré une antibiothérapie correcte, les cas avancés présentent souvent une issue fatale. Une intervention chirurgicale rapide est de plus souvent nécessaire, ce qui accroît l'importance d'un diagnostic précoce.

\section{Mycobacterium chimaera: qu'est-ce?}

$M$. chimaera est une mycobactérie non tuberculeuse à croissance lente, formant un biofilm. Elle a été découverte en 2004 et fait partie du complexe Mycobacterium avium. Son identification nécessite des examens microbiologiques particuliers. C'est une cause rare de pneumonie chez les patients immunosupprimés. Ce n'est que suite à l'identification de cas décrits plus haut que son implication dans des cas d'endocardites, d'infections de prothèses vasculaires et d'infections disséminées a été découverte.

\section{Quand doit-on penser à une infection M. chimaera?}

Selon les connaissances scientifiques actuelles, il faudrait suspecter une infection à $M$. chimaera chez tous les patients présentant une infection d'origine indéterminée, une endocardite à hémoculture négative ou des symptômes de maladie systémique des mois ou des années après une intervention chirurgicale nécessi- 
tant l'utilisation d'une machine cœur-poumon. Sont avant tout concernés les patients ayant bénéficié de la pose d'une valve cardiaque artificielle, d'une prothèse vasculaire ou d'une pompe mécanique intracardiaque. Toutefois quelques rares cas d'infections à $M$. chimaera ont été observés chez des patients après une opération avec HCDs sans implantation de matériel étranger (pontage aorto-coronarien). Le lien avec des HCDs contaminés en cas de transplantation pulmonaire est encore incertain.

\section{Mesures concrètes}

On peut présumer que l'infection ne concerne qu'un nombre très limité de patients. La valeur prédictive négative des méthodes diagnostiques dans la phase latente est basse. Un examen de grande envergure de tous les patients n'est donc pas judicieux. Lors de situations bien définies (voir plus bas), de fièvre d'origine indéterminée ou d'une maladie systémique indéterminée chez des patients après une opération avec HCDs, même plusieurs années auparavant, ce diagnostic doit être exclu - en particulier, si en cas de suspicion d'endocardite, les hémocultures conventionnelles restent négatives.

\section{Quand doit-on suspecter une infection}

\section{à $M$. chimaera et adresser le patient}

\section{à une consultation spécialisée d'infectiologie?}

Si le critère d'exposition et un ou plusieurs points cidessous sont remplis, il est recommandé d'adresser ces patients à une consultation spécialisée d'infectiologie pour de plus amples investigations.

Chirurgie cardiaque avec circulation extracorporelle et utilisation d'un Heater Cooler Device (valves artificielles, o prothèses vasculaires, transplantation cardiaque, pompe mécanique intracardiaque, pontage aorto-coronarien) dans n'importe quel hôpital en Suisse

et un/plusieurs des points suivants

Correspondance: PD Dr. med. B. Hasse Klinik für Infektionskrankheiten und Spitalhygiene UniversitätsSpital Zürich

Rämistrasse 100

CH-8091 Zurich

Tél. 0442559237

barbara.hasse[at]usz.ch

Infection de prothèse aortique thoracique à hémocul.

tures négatives

o Sternite et/ou médiastinite à hémocultures négatives

o Fièvre d'origine indéterminée ou vasculite

o Maladie systémique indéterminée

- Sarcoïdose ou autre maladie granulomateuse

\section{Investigations recommandées en cabinet}

- Anamnèse (fièvre, fatigue, perte de poids, sueurs nocturnes)

- Examen physique

- Laboratoire (vitesse de sédimentation, protéine C réactive, formule sanguine, lactate déshydrogénase [LDH], transaminases, créatinine)

- Hémocultures

\section{Examens en milieu spécialisé}

- Echocardiographie

- Histopathologie en cas de réopération / biopsie cardiaque

- Cultures mycobactéries/PCR:

- Hémocultures mycobactéries (BacTec myco Lytic/ F bottles [BD Bioscience])

- Cultures mycobactéries ou PCR mycobactéries en cas de réopération / biopsie

\section{Informations complémentaires}

Office fédéral de la santé publique OFSP

Division Maladies transmissibles

Tél. +41 (0)58 4638706

epi[at]bag.admin.ch

\section{Références}

1 Sax H, Bloemberg G, Hasse B, et al. Prolonged Outbreak of Mycobacterium chimaera Infection After Open-Chest Heart Surgery. Clinical infectious diseases: an official publication of the Infectious Diseases Society of America 2015;61(1):67-75.

2 Massnahmen für höhere Patientensicherheit in der Herzchirurgie. Bundesamt für Gesundheit (14.07.2014). https://www.news.admin. $\mathrm{ch} / \mathrm{message} /$ index.html?lang $=$ de\&msg-id $=53774$

3 Hypo-/Hyperthermiegeräte (Heater Cooler Units, HCU) für die Herzchirurgie - neue Empfehlungen: Mögliche Beeinträchtigung von Oxygenatoren. Swissmedic (21.06.2016). Hypo-/Hyperthermiegeräte (Heater Cooler Units, HCU) für die Herzchirurgie - neue Empfehlungen - Swissmedic.

4 Risk assessment on Mycobacterium chimaera infections associated with heater-cooler units. ECDC (18.11.2016). http://ecdc. europa.eu/en/publications/Publications/RRA-mycobacteriumchimaera-November-2016.pdf 